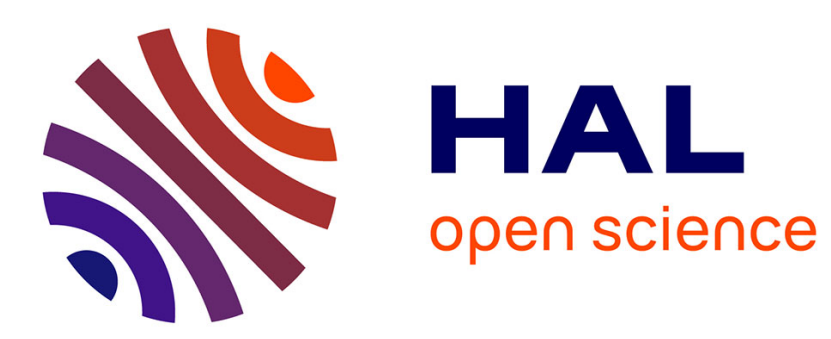

\title{
Soins précoces : devenir psychosocial des familles et développement des enfants
}

\author{
Annick Camille Dumaret, Virginia Picchi
}

\section{To cite this version:}

Annick Camille Dumaret, Virginia Picchi. Soins précoces: devenir psychosocial des familles et développement des enfants. Annales Médico-Psychologiques, Revue Psychiatrique, 2005, 163 (6), pp.476-485. halshs-00353174

\section{HAL Id: halshs-00353174 \\ https://shs.hal.science/halshs-00353174}

Submitted on 14 Jan 2009

HAL is a multi-disciplinary open access archive for the deposit and dissemination of scientific research documents, whether they are published or not. The documents may come from teaching and research institutions in France or abroad, or from public or private research centers.
L'archive ouverte pluridisciplinaire HAL, est destinée au dépôt et à la diffusion de documents scientifiques de niveau recherche, publiés ou non, émanant des établissements d'enseignement et de recherche français ou étrangers, des laboratoires publics ou privés. 


\section{SOINS PRECOCES : DEVENIR PSYCHOSOCIAL DES FAMILLES \\ ET \\ DEVELOPPEMENT DES ENFANTS}

\section{Dumaret Annick-Camille $^{1}$ et Picchi Virginia ${ }^{2}$}

${ }^{1}$ Psychologue, ingénieur de recherche à l'INSERM,

Centre de Recherche Médecine, Sciences, Santé et Société (CERMES)

Site CNRS, 7 rue Guy Môquet, 94801 Villejuif Cedex

${ }^{2}$ Psychologue, Centre Alfred Binet, 76 Avenue Edison, 75013 Paris.

Auteur/correspondance:

A.C. Dumaret, Ingénieur de recherche à l'INSERM, CERMES, INSERM U.502 Adresse : site CNRS, 7 rue Guy Môquet, 94801 Villejuif Cedex

Tel : 01.49.58.35.91 Fax : 01.49.58.34.38

Courriel : dumaret@vjf.cnrs.fr

Titre abrégé : Soins précoces et devenir des familles 


\section{Résumé :}

Le devenir de familles fragilisées par des difficultés psycho-affectives et/ou psychiatriques suivies très précocément a été étudié cinq ans au moins après la sortie d'une institution soignante. L'action thérapeutique avait porté sur l'enfant, âgé de moins de dix huit mois à l'admission, sa mère, le lien enfant-parent(s) et l'environnement socio-familial. Trente et une situations ont été réévaluées à partir d'entretiens semi-directifs et d'outils standardisés. Cet article est centré sur la situation actuelle des familles, leurs compétences socio-relationnelles et parentales et sur l'adaptation des jeunes à travers leurs compétences intellectuelles, socioscolaires et comportementales. Aujourd'hui, la parentalité est assumée, même si elle reste partielle dans le cas des parents ayant des enfants placés. Aucune maltraitance grave n'a été repérée dans les familles et la situation des enfants a évolué de façon positive. Les soins précoces de longue durée ont joué un rôle médiateur par rapport aux facteurs de risque psychosociaux des familles.

Mots clé : soins précoces, familles à risque, parentalité, scolarité, comportements

Titre en anglais : Early intervention: psychosocial outcome of families and children's development

\section{Summary:}

This research reports the outcome of families whose children were followed in an outpatient treatment centre before the age of eighteen months and who had been out of treatment for at least five years. Families exhibiting psycho-emotional and/or psychiatric problems were treated for a period of three to six years. Thirty-one situations were re-evaluated using standardized materials and semi-structured interviews with parents, children, and foster families. Results focus on: 1) the families' present situation, their social/relational and parental competence; 2) the intellectual, social/scholastic and behavioural competence of the young people. Even for children in care, parents are able to carry out their responsibilities. No serious child abuse or neglect was detected and there was a positive evolution for the majority of the children. Given these results, we can assume that early therapeutic intervention mediates psycho-social risk in these families.

Keywords: families at risk, early intervention, parenthood, outcome, child development 


\section{Résumé long en anglais}

In the past decades, an extensive body on early intervention research programs has grown with numerous scientific publications in the English language litterature, while such studies remain rare in France. This study reports the outcome of families whose children were followed in an out-patient treatment centre, the Unité de Soins Spécialisés à Domicile (USSD) in Paris. This institution, created in 1975, was designed to promote healthy parent-infant relationships and prevent difficulties for families exhibiting psycho-emotional and or psychiatric risks. The objectives are to enhance infant and early child development, to support parenting competences and to fight against intergenerational process of maltreatment and child placement. The theoretical references of the multidisciplinary team are the psychanalyse, the attachment theory and the clinic of interactions.

Thirty-height families were selected from the files of the USSD: They all had a child aged before the age of 18 months, born between 1985 and 1990 and were cared for more than one year; the other children received intervention after this age. Assessment of parents and children was made when the families had been out of treatment for at least five years. As it is a preliminary study without a control group, we have choose a psychosocial and comprehensive approach more centered on family issues than individual ones. For each child and family, institutional data were collected and argued with the all the professionals still present in the institution: admission in the institution, parental problems and infant symptoms (with reference to the DSM IV and the Zero-to-Three classification), therapeutic interventions (psychodynamic or interpersonal, parents-children groups, parental or children groups, home visiting, ...), child's re-educations, relationship with other social or psychiatric teams, orientation after the intervention.

Thirty-one situations were re-evaluated. Standardized materials (WISC-R subtests, Rutter A scale for children, Nottingham Health Profile for parents) and semi-structured interviews were used for the assessments. Parental consent and administrative authorizations (for children in foster families or institutions) were obtained and interviews were registered.

Two groups of families were studied. Group I included families without specific pathology, they received therapeutic intervention for a mean of $21 / 2$ years, most of the mothers were depressed. It was composed of 2 sub-groups : in 8 families, the child's birth with severe perinatal problems had brought about the parental trauma, for 7 families, psycho-emotional problems were blew up again after the child's birth, signs of maltreatment could be detected. Group II included families at high risk for child abuse and neglect with numerous psychosocial stress, most of them were followed by social workers; interventions had lasted for a mean of 6 years. It was composed of 2 subgroups: 7 disorganized family situations with alcohol abuse and depressed mothers, and 9 families with psychotic mothers, most of the babies were placed in foster care.

We met 16 parental couples and 13 monoparental families twice and 7 foster families. Twenty five young people were interviewed and complementary information was obtained for seven others from the Child Protection services and foster agencies.

Results focus on: 1) the families' present situation, healh representations and their social/relational and parental competences ; 2) for the young people: the intellectual and scholastic competences, behaviour, social relationships with their peers. They are presented in each of the sub-groups for parents and for children ; differences and common points are noted in the two groups of families I and II. Mean ages at assessment were 12,3 years for children in group I and 13,3 in group II. 
Except in one case (children became Wards of the State), parents are able to carry out their responsibilities, totally in group I or partially in group II where it was shared with the Child Protection services for most children and adolescents were still placed. No serious child abuse or neglect was detected and there was a positive evolution for the parents' social and family relationships especially in group I. The family support is more precarious in group II, counterbalanced by social and medical support. Concerning children and adolescents, two third are in the normal school system, the others are in classes for slow learners, one is in a day care hospital. A third have behaviour problems. For all of these young people, verbal subtests (Vovabulary and Similarities were more succeeded thant than the performance subtest (Block Design), this result is associated with facilities in verbal expression showed during the interviews. The impact of early therapeutic intervention is notable among children of the families at high risk who had received such care befor the age of one: the all have better social competences with peers, less behaviour problems and school failure than the others.

Given these results, we can assume that early therapeutic intervention mediates psycho-social risk in these families. As this study is mostly descriptive, it should be followed by complementary research on larger populations. 
Sous l'essor de la psychanalyse et de la psychiatrie du nourrisson, les soins et traitement du bébé et de son environnement se sont développés. Ainsi, l'Unité de Soins Spécialisés à Domicile de jeunes enfants du XIII ${ }^{\text {ème }}$ arrondissement de Paris, a été créée en 1975 par Myriam David pour faciliter l'accès aux soins à des familles à risque où se conjuguent les difficultés d'ordre psychopathologique et social. Elle n'est pas une unité d'hospitalisation mère-enfant mais c'est le seul service d'éducation spécialisée et de soins à domicile psychiatrique de Paris. Après plus de vingt ans de fonctionnement, il y avait lieu de questionner l'impact à moyen terme des soins précoces sur le développement du nourrisson à haut risque et le devenir de ses parents.

Dans le cadre des interventions précoces, on note deux mouvements importants, celui de la psychiatrie du nourrisson ${ }^{1}$ et celui de la pédiatrie sociale. Le travail en réseau, qui développe les liens entre les équipes de la petite enfance et les équipes de soins psychiatriques, articule ces différents courants et a un impact préventif certain. Des programmes de soins ont été mis en place avec des interventions à domicile ou sur les lieux de vie fréquentés par les petits enfants. Les écrits témoignent souvent à partir de méthodes rétrospectives du développement du petit enfant eu égard à la psychopathologie parentale $[8,36]$. Les prises en charge précoces axées sur les dyades mère-enfant selon le modèle thérapique de Fraiberg, ont montré des résultats positifs quant à l'amélioration de la situation des mères (diminution des états dépressifs, amélioration des interactions mère-enfant) et celle des enfants, notamment un meilleur développement intellectuel vers l'âge de deux ans ([1, 27]. Les évaluations à la fin des 
soins ou à plus long terme par des pédiatres et des psychologues indiquent une diminution de la maltraitance $[2,4,30]$. D’autres travaux ont été publiés sur les interventions thérapeutiques brèves et l'évolution des enfants à la préadolescence [6]. Si les évaluations de prises en charge précoces de type médicosocial, psychologique ou psycho-éducatif ont fait l'objet de très nombreuses publications scientifiques en langue anglaise [9], actuellement en France, on ne dispose pas d'étude sur les effets à long terme de ces prises en charge.

\section{Le contexte institutionnel de la recherche}

L'action thérapeutique de 1'Unité de Soins Spécialisés à Domicile pour jeunes enfants concerne l'enfant, sa mère, le lien enfant-parent(s) et l'environnement sociofamilial [7]. Les demandes de soins sont majoritairement effectuées par des travailleurs médico-sociaux, les familles étant adressées par la PMI, l'ASE et des équipes périnatales. Il s'agit de permettre les soins propices au développement du petit enfant en repérant ses modalités défensives [22, 25]. Il s'agit aussi de soutenir l'accès à la parentalité, et de lutter contre les processus intergénérationnels qui induisent maltraitance et placements. Quant l'enfant est placé pendant la prise en charge, le traitement vise à ce que l'indication de placement soit perçue comme un soin par les parents et non comme une sanction [21].

Le traitement dit «direct» auprès du bébé et de ses parents intervient quand la famille accepte de reprendre à son compte la demande du professionnel : «C'est l'enfant qui est la cause de la rencontre, et c'est pour lui que les familles feront plus que ce qu'elles ne feraient pour elles-mêmes » [Fraiberg, 13]. Le traitement dit

${ }^{1}$ Les professionnels de ces diverses disciplines se sont fédérés dans le cadre de 1'Association mondiale pour la santé mentale du jeune enfant (WAIMH, groupe francophone créé à Paris 
«indirect» concerne le soutien aux équipes de professionnels du réseau qui peuvent être mis à mal dans leur travail avec des familles dont les modes relationnels sont très désorganisés. C'est par le travail en réseau que ces parents, non demandeurs de soins, peuvent être approchés. Parallèlement aux traitements habituels de type psychothérapique et aux rééducations, des outils spécifiques de soin ont été développés : l'observation du bébé dans ses lieux de vie, en référence aux travaux d'E. Pickler et d'E. Bick, le travail à domicile, les accompagnements thérapeutiques, les groupes à visée thérapeutique, etc. L'équipe est pluridisciplinaire, ses références théoriques sont la théorie psychanalytique et celle de l'attachement avec la clinique des interactions.

C'est une population hétérogène qui a été étudiée, répartie a priori en deux grands profils : a) des familles sans psychopathologie installée (I), avec celles où les enfants présentaient un handicap ou des problèmes périnataux (HAND) et celles ayant des troubles de la parentalité (PAR), et b) des familles à haut risque (II) du fait de difficultés psychosociales nombreuses (carences multiples, CAR) et ou de troubles psychiatriques maternels (psychose chronique, PSYK).

\section{MATERIEL ET METHODE}

\section{Hypothèses de la recherche}

Sachant que les signes de souffrance et les modalités défensives du bébé passent facilement inaperçus au cours de la première année pour devenir plus manifestes, voire être fixés plus ou moins gravement ensuite, ces soins précoces ont-ils permis un développement « dans la norme » de ces enfants quant à leur croissance physique, leurs capacités affectives et cognitives et leur organisation psychique? Ont-ils permis 
d'éviter le risque de pathologie déficitaire ou mentale et également de rompre avec la répétition de la maltraitance? On s'est aussi interrogé sur le rôle du support familial et social. Plusieurs auteurs ont montré l'importance de ce facteur dans la prévention des troubles psychiques chez l'enfant [3, 34]. Quand ce support est perçu comme faible, les enfants présentent plus de troubles de comportement, leur agressivité témoignant de leur sentiment d'impuissance et de leur difficulté à gérer les relations avec les autres [23]. Le lien entre support social, santé et bien-être des adultes étant reconnu, on a étudié l'état de santé général des parents, santé entendue au sens large de la définition de l'OMS en 1946.

\section{Sélection de la population, aspects déontologiques}

Trois critères de sélection de la population à partir des registres d'admission de l'institution ont été décidés : 1) une prise en charge avant l'âge de 18 mois ,2) un suivi thérapeutique d'au moins un an et, 3) une date de naissance entre 1985 et 1990 : ceci permettait d'évaluer plus de 5 ans après la sortie de l'institution des enfants d'âge scolaire. Un courrier du médecin-directeur de l'Unité de Soins a été adressé aux familles. Certaines n'y répondant pas, une ancienne professionnelle de l'Unité les a recontactées en se déplaçant à leur domicile. Pour rencontrer les enfants placés à l'ASE, les autorisations ont été demandées aux parents; elles ont également été demandées aux autorités administratives de l'ASE afin de pouvoir interviewer leurs familles d'accueil (FA). La confidentialité des données recueillies avait été garantie aux familles et l'ensemble du protocole de recherche a reçu l'aval de la Commission Nationale Informatique et Libertés en 1998.

\section{Collecte des données et analyses}

\subsection{Dépouillement du dossier institutionnel}


Une grille a été élaborée et discutée avec les professionnels de l'institution, en référence au DSM-IV et à la classification diagnostique Zero-to-Three. Pour chaque enfant, les données ont été retranscrites par deux cotateurs : processus d'admission et difficultés parentales, symptomatologie de l'enfant, prises en charge et travail avec l'environnement, arrêt des soins et orientation à la sortie de l'institution. Pour chaque situation, une synthèse individuelle a été rédigé par l'équipe de recherche ; l'ensemble a été revu avec les différents soignants ayant suivi les familles.

\subsection{Protocole d'enquête}

Les évaluations ont été effectuées par deux psychologues extérieures à l'institution : l'une centrée sur les adultes (deux entretiens avec les parents élevant leurs enfants, un seul pour ceux dont les enfants étaient placés et pour les familles d'accueil), l'autre centrée sur les enfants (un entretien). Aucune famille n'a refusé l'enregistrement au magnétophone des entretiens, réalisés entre 1999 et 2001.

- Données sur les familles : les thèmes abordés concernaient la situation de la famille, son évolution depuis la fin des soins à l'Unité, les relations parents-enfants, leurs représentations de l'enfant, les identifications familiales... Les différents axes de la parentalité ont été repérés: son exercice, son expérience et sa pratique [18]. L'état de santé a été évalué à partir du discours des parents et confronté aux données d'un questionnaire, le Nottingham Health Profile NHP [13].

- Données sur les jeunes: L'entretien était centré sur la vie quotidienne et leurs représentations d'eux-mêmes et de la famille. La scolarité de chacun a été reconstituée. Trois évaluations ont été effectuées : a) une estimation du quotient intellectuel $\mathrm{QI}^{2}$ à partir de trois subtests du WISC-III : Vocabulaire, Similitudes et Cubes, b) une évaluation des troubles du comportement (questionnaire A de Rutter ${ }^{3}$ rempli par les parents ou la FA) et c) une estimation des compétences sociales avec les pairs à partir des entretiens des enfants et des adultes (tableau III). Le protocole a prévu aussi une étude de leur fonctionnement psychique au travers de l'analyse de leur discours à partir de trois histoires à raconter ; cette analyse permet de repérer la

${ }^{2}$ Une estimation du QI peut être effectuée à partir de plusieurs subtests [16]. Nous avons vérifié ce lien entre subtests et note globale à partir d'un échantillon de 90 jeunes de 10-15 ans ayant passé tout le WISC-III (communication personnelle, Marie Claude Chivot, psychologue, Pontoise) : les différences observées entre QIG moyen $(109,7 \pm 18,2)$ et QI estimé $(110,9 \pm$ $17,2)$ ne montrent pas d'écart significatif.

${ }^{3}$ Cette échelle (31 items) permet de relever les problèmes de comportement (score $\geq 13$ ), avec trois scores : névrotique (anxiété, troubles internalisés), anti-social et hyperactif. 
façon dont ils se perçoivent en tant que sujets et d'appréhender leur capacité d'empathie et de réflexion. Pour les jeunes non interviewés, les référents des placements et les assistantes maternelles ont été rencontrés.

\subsection{Analyse des données}

Les données ont fait l'objet d'une quantification des évaluations standardisées pour chaque groupe d'enfants étudié (deux cotateurs) avec des comparaisons inter-groupes (analyse de variance, tests non paramétriques: Kruskal-Wallis et Mann-Whitney). L'analyse clinique des entretiens, famille par famille (parents, enfants et parfois assistantes maternelles et référents sociaux) a été discutée au sein de l'équipe de recherche. Les informations sur la situation actuelle ont été confrontées aux synthèses individuelles, aux grilles institutionnelles et à la connaissance des situations par l'un des cliniciens, médecin-directeur de l'institution.

\section{Description des situations familiales étudiées}

Le tableau I résume les principales caractéristiques des enfants repérées dans les premières semaines de l'admission à l'Unité (classification 0-3 ans), la durée moyenne des soins et l'âge moyen à l'évaluation.

Le groupe des familles sans pathologie définie (I): les mères et quelques pères, présentaient des états dépressifs, réactionnels au traumatisme de la naissance ou liés à la réémergence de conflits internes anciens. Les soins ont commencé avant l'âge d'un an pour la moitié des 16 enfants; parents et enfants ont tous été suivis environ entre deux et trois ans.

- groupe HAND (huit familles) : après l'annonce du handicap, la moitié avaient demandé une aide auprès d'un psychiatre. La relation parents-enfant était sous impliquée ou anxieuse.

- groupe PAR (sept familles, l'une a deux enfants) : les mères étaient dans un état dépressif important parfois aigu, l'une présentait en plus des troubles de la personnalité de type borderline. Les troubles des enfants étaient en lien avec la relation maternelle; des signes de risque de maltraitance d'ordre psychologique, pouvaient être perçus. 
Les familles à haut risque (II) : on est face à des situations familiales désorganisées, marquées par de nombreux traumatismes antérieurs (deuils, ruptures...), sources de graves négligences et de risques de maltraitance; les besoins vitaux des enfants n'étaient pas assurés ou donnés de façon discontinue, le développement somatopsychique était à risque. Les stress psychosociaux étaient présents dans toutes ces familles dont la plupart ont fait l'objet de suivis sociaux ou judiciaires. Les soins ont commencé avant l'âge d'un an pour 11 des 16 enfants et ont duré en moyenne six ans pour tous.

- groupe CAR (sept familles) : la psychopathologie maternelle principale était la dépression pour cinq mères, quatre mères étaient consommatrices d'alcool et 4 mères présentaient des états limites. Un diagnostic spécifique de troubles avait été posé pour tous les bébés sauf un. Trois enfants avaient déjà passé plus de temps à 1'hôpital ou en placement qu'en famille.

- groupe PSYK (neuf familles): toutes les mères sauf une étaient suivies en psychiatrie; quatre pères avaient un diagnostic psychiatrique. La plupart des bébés n'étaient plus dans leur famille (pouponnière, unité hospitalière mère-enfant); les troubles significatifs concernaient la relation parents-enfant.

\section{Tableau I}

Lors des évaluations, nous avons rencontré : 1) seize couples et treize mères seules, sept assistantes maternelles ; 2) vingt cinq jeunes ; pour les sept autres, des informations de plusieurs sources ont été obtenues (mère, assistante maternelle, référents institutionnels), conférant une validité à leur contenu. Parmi ces jeunes, deux adolescents venaient de changer de type de placement à l'ASE, il nous a paru non judicieux de les rencontrer. Soulignons que plusieurs familles connaissent le langage des équipes psychiatriques, sociales, judiciaires, ce qui a pu se traduire par une apparente adhésion aux propos des interlocuteurs rencontrés.

\section{RESULTATS}


Cet article présente la situation psychosociale actuelle des familles, celle des parents (milieu socio-familial, santé, parentalité) et celle de leurs enfants (situation socio-scolaire et comportementale), en repérant les caractéristiques communes à chaque type de familles, puis les sous-groupes avec leurs spécificités.

\section{LES FAMILLES SANS PSYCHOPATHOLOGIE DEFINIE}

\section{Le milieu parental}

\section{1. données socio-démographiques, relations familiales, santé}

Parmi ces parents, on dénombre treize couples stables (12 vivent toujours ensemble depuis plus de dix ans) ; tous les enfants sont légitimes ou reconnus par leurs deux parents à l'exception de ceux de deux mères célibataires. Seules quatre familles n'ont qu'un enfant, la plupart en ayant eu d'autres au décours du suivi à 1'Unité. La moitié de ces parents ont un niveau d'études supérieur ou égal au baccalauréat. La vie professionnelle est très investie, ainsi toutes les mères PAR travaillent à temps plein. Les relations familiales et fraternelles des parents PAR sont plus complexes que celles des familles HAND - par exemple les grands-parents sont moins présents et aidants - et plus marquées par des conflits, qui n'ont toutefois plus aujourd'hui la même importance qu'autrefois. En effet, les parents ont généralement renoué des liens avec la parentèle, les mères célibataires PAR se sont efforcées, au delà de leurs déceptions personnelles, de maintenir la place du père auprès de leur(s) enfant(s).

Ces parents disent avoir une bonne santé, il n'y a ni suivi psychiatrique, ni prise de médicament. Deux mères PAR ont maintenu un suivi psychothérapique. Les résultats du questionnaire de santé confirment leur discours. Comparativement aux données du NHP, ce sont les mères PAR qui ressemblent le plus à la population générale; pour les mères HAND et également les pères, l'écart des résultats avec les scores moyens du 
NHP fait penser que ces parents sous-estiment leurs propres difficultés de santé, trop préoccupés qu'ils sont par l'état de santé de leur enfant.

\subsection{Les relations parents-enfants}

Lors des entretiens, les parents ont pu évoquer la souffrance éprouvée au cours des premiers mois de l'enfant et leurs difficultés relationnelles à investir cet enfant-là. Leur capacité à parler du passé et des contraintes actuelles vécues au quotidien témoigne de l'élaboration du traumatisme, ils ont acquis une possibilité de distanciation. Aujourd'hui, la parentalité, construite dans la continuité du quotidien, est assumée sans déni de la souffrance passée; les relations parents-enfant se caractérisent par un investissement à dominante objectale. Tout en maintenant une vie de couple, ces parents s'efforcent d'apporter sécurité et confiance à leurs enfants. Ils dialoguent avec eux, donnent beaucoup d'importance aux échanges directs, négocient et réaménagent parfois leurs positions, sont capables d'autocritique; ils acceptent la progression vers l'autonomie de leurs enfants et sont soucieux de leur avenir. Attentifs à leur bien-être, ils anticipent certaines difficultés de leurs enfants et leurs besoins sont entendus, comme en témoigne l'acceptation des parents de demandes de consultation thérapeutique récentes de certains de leurs enfants. On a noté que dans les familles HAND, il reste des traces de surprotection dans la relation mère-enfant, même s'il n'y a pas de dépendance physique ni séquelle grave.

\section{Les enfants de ces familles}

Tous ont été rencontrés. Dans l'ensemble, leur insertion familiale et scolaire ainsi que leurs relations avec leur entourage et leurs pairs sont satisfaisantes. Cependant, plusieurs ont fait état de sentiment de vécu de rejet ou d'isolement dans le passé, qui persiste encore aujourd'hui. La façon dont ils parlent de leur handicap, de leur petite enfance et d'eux-mêmes reflète de capacités d'introspection et d'élaboration. 
2.1. Les jeunes ayant subi des accidents périnataux ou présentant des atteintes génétiques $(\mathrm{n}=8)$

Ces problèmes (tableau I) n'ont pas empêché l'insertion de la majorité d'entre eux dans leurs milieux de vie. Pour quatre jeunes, des atteintes sont irréversibles sur le plan neurologique, moteur et/ou cognitif et de langage (deux jeunes filles se déplacent en fauteuil roulant), un adolescent présente un syndrome de Pierre Robin et une trisomie 8. A l'exception de trois d'entre eux en éducation spéciale, les autres n'ont aucun retard scolaire ; leur niveau intellectuel est légèrement inférieur à ce qui serait attendu de par leur milieu social (tableau II). Trois jeunes ont des troubles de comportement, de type névrotique (tableau III). Il semble persister des troubles relationnels sous des formes variées pour plusieurs jeunes. Par exemple, une relation empreinte d'adhésivité persiste chez une jeune fille alors que le retrait émotionnel et l'attachement inhibé ont disparu. Pour un garçon, le repli autistique a été levé ; anxieux, il se perçoit comme différent des autres, sa mère le dit très collé à elle.

\subsection{Les jeunes de parents présentant des troubles de la parentalité $(n=8)$}

D'un bon niveau intellectuel, ils n'ont aucun retard scolaire à l'exception d'un adolescent en hôpital de jour. Trois jeunes ont des difficultés comportementales dont deux de type névrotique et un de type antisocial avec hyperactivité.

A la différence du groupe HAND où les références identificatoires se trouvent essentiellement au sein de la famille, ces jeunes ont des relations plus larges avec l'entourage amical. Ils évoquent facilement leurs difficultés relationnelles avec leurs parents, les critiquent et s'opposent - ce qui étant donné leur âge, peut être considéré comme adapté. Pour certains, des difficultés repérées dans la petite enfance et à la fin des soins paraissent en lien avec celles présentes lors de nos rencontres : 1) une fillette de dix ans, sociable et créative, a toujours des problèmes d'attention, ses troubles de 
comportement sont de type oppositionnel et hyperactif; 2) l'inhibition relationnelle d'un préadolescent a été mise en relation par sa mère avec ses propres difficultés vécues à la naissance et non parlées avec son fils (bébé confié en vue d'adoption et repris par la mère dans le délai de rétractation) ; 3) le jeune suivi en hôpital de jour pour autisme présente encore une pathologie importante, il a conscience de ses problèmes relationnels.

\section{LES FAMILLES A HAUT RISQUE}

\section{Le milieu parental}

\section{1. données socio-démographiques, support social et santé}

Une certaine stabilisation des situations s'est installée au fil des ans dans la vie conjugale (les six couples parentaux se sont maintenus) et quant au lieu de vie. Les familles vivent en majorité de prestations sociales et familiales (AAH, RMI...). Ces parents ne peuvent compter sur un entourage familial aidant, le faible réseau de sociabilité explique l'importance du support socio-médical : l'interlocuteur privilégié reste un travailleur social connu de longue date ou un médecin. A l'époque des entretiens, la violence et les risques de maltraitance semblent avoir disparu. Le groupe CAR est composé surtout de familles monoparentales $(5 / 7)$ où les pères sont à distance. La majorité des fratries (de 1 à 4 enfants) sont de pères différents. Ces familles sont en majorité issues de milieu ouvrier et rural, le niveau d'études des parents dépasse à peine le début du secondaire, deux mères sont illettrées ; seules deux mères travaillent. Dans le groupe PSYK : on dénombre cinq mères seules et quatre couples parentaux. Les familles sont issues de milieux variés, le niveau d'études va du primaire à l'enseignement supérieur. La moitié des mères ont ou ont eu une activité professionnelle. Alors que deux mères du groupe CAR ont eu un autre enfant au 
décours des soins, on constate qu'aucune naissance n'est survenue dans le groupe PSYK depuis la sortie des soins.

La vie quotidienne de toutes ces familles est dominée par des problèmes de santé physique et mentale. L'état général de santé des mères CAR est très déficient (conduites addictives, vieillissement prématuré...): quatre sont suivies par un généraliste et prennent des médicaments psychotropes, les trois autres sont hors de tout système de soins. Toutes les mères PSYK sont toujours suivies en psychiatrie, elles ont accepté leur «statut de patient»; selon leurs dires confirmés par les informations recueillies auprès d'équipes sociales, la moitié n'ont pas été réhospitalisées en psychiatrie pendant les cinq ans écoulés depuis la sortie de l'Unité. Trois sont sous tutelle. Quatre d'entre elles s'appuient sur un compagnon souvent luimême non exempt de troubles pathologiques. Le questionnaire NHP confirme cet état de santé déficitaire par rapport à la population générale ; deux registres différents sont mentionnés, l'évocation importante de la douleur chez les mères carencées, laquelle s'associe à un état dépressif chronique, et l'isolement relationnel qui domine chez les mères psychotiques.

\subsection{Une fonction parentale souvent partagée}

En dépit de différences individuelles, la majorité de ces mères s'inscrivent dans une histoire familiale dont elles peuvent évoquer des souvenirs, mêmes pénibles. Elles revendiquent une image de mère qu'elles ont construite au fil des années. Les familles qui élèvent leurs enfants sont suivies en AEMO. Mais dans les trois-quarts des situations, les enfants vivent en placement $(n=11)$, il s'agit surtout des enfants de mères psychotiques ( $8 / 9$ vs $3 / 7$ pour les enfants de mères carencées). Ces placements sont stables : sept jeunes sont toujours dans les mêmes familles d'accueil, deux adolescents qui viennent d'intégrer un foyer gardent un lien avec leur FA, une seule fillette a vécu 
un échec de placement en FA. Une enfant et sa mère ayant une pathologie psychotique ont vécu ensemble dans une FA pendant plus de dix ans; actuellement la jeune fille vit dans un foyer et sa mère vit désormais seule dans un appartement thérapeutique. Les parents rencontrent très régulièrement leurs enfants. L'évocation du placement réactive toutefois souffrances et revendications pour ces parents.

\subsection{Les relations parents-enfants}

Après une prise en charge thérapeutique d'un premier enfant à l'Unité de Soins, la nécessité d'un suivi s'est imposée à l'arrivée d'une nouvelle naissance dans quatre familles carencées. Elle a été réalisée avec plus de souplesse entre les parents, l'enfant et l'équipe soignante. Ces mères soulignent que ces jeunes suivis très tôt ont moins de troubles de comportement et sont plus faciles à vivre que leurs aînés. Elles-mêmes sont en difficulté quand il s'agit de trouver des compromis dans les relations avec leur(s) enfant(s), souvent idéalisé(s). Leur investissement parental est à dominante narcissique et les séparations avec leurs enfants adolescents ou adultes sont toujours source de souffrance.

Les mères psychotiques se montrent lucides quant à leurs propres difficultés et à leurs manques, certaines sont soulagées de savoir leur enfant à l'abri de leur état de santé. Elles reconnaissent la souffrance de leur enfant aujourd'hui différencié d'ellesmêmes, même si leur investissement reste encore empreint d'imaginaire, avec des interactions houleuses et des manifestations de sentiments excessives.

\section{Les jeunes des familles à haut risque}

Seule la moitié des jeunes ont pu être rencontrés. Ceux qui vont le mieux aujourd'hui ont un bon niveau intellectuel, font des activités sportives ou musicales, ont investi leurs frères et sœurs, leurs pairs, copains ou autres enfants de la famille d'accueil (tableaux II et III) et font également des projets pour l'avenir. Ils bénéficient 
d'un accompagnement très présent par le biais d'une personne privilégiée (la référente du placement) ou de la continuité d'un investissement affectif au sein de leur famille. Aujourd'hui, pour la moitié de ces jeunes, les soins psychothérapeutiques se poursuivent ou ont repris à leur demande. L'angoisse d'abandon ou de séparation est présente, plus nuancée pour ceux arrivés tardivement en FA, familles qui les avaient déjà accueillis auparavant pour des séjours vacances lors de la prise en charge à l'Unité.

\subsection{Les enfants de familles carencées $(n=7)$}

Pour les trois jeunes testés, le QI est proche de la moyenne. Quatre parmi les sept poursuivent une scolarité normale avec au maximum un an de retard, les autres sont en échec scolaire (deux en SEGPA) et présentent des troubles comportementaux plus ou moins importants. L'un d'eux, un adolescent présentant un niveau intellectuel proche de la moyenne, a des troubles manifestes de type anti-social et hyperactif; on note qu'il a demandé à reprendre des séances de psychodrame dans l'institution qui le suivait. Les deux autres vivent avec leur(s) parent(s) ; 1'école n'est pas une priorité pour les mères qui ne font pas de projet d'avenir pour leur enfant : 1) pour la fillette, le déficit cognitif peut être corrélé avec un syndrome d'alcoolisation fœetale et avec les limitations intellectuelles parentales ; 2) le garçon a souffert d'un investissement chaotique lié à l'état défaillant de sa mère et à ses problèmes de santé (trois hospitalisations dans sa première année de vie).

\subsection{Les enfants de mères psychotiques $(n=9)$}

Les résultats aux subtests de vocabulaire et de similitudes indiquent que ces jeunes présentent un niveau intellectuel proche de la moyenne mais sont en échec au subtest des cubes, échec qui reste inexpliqué. Six jeunes suivent une scolarité sans retard 
important, les trois autres sont en échec ( 2 ans de retard, EREA, SEGPA). Quatre présentent des troubles du comportement: troubles de type névrotique chez deux adolescents et de type antisocial pour le troisième, troubles indifférenciés chez la seule enfant vivant en famille. Par ailleurs, plusieurs jeunes présentent des symptômes somatiques (alimentation, sommeil). Leur fonctionnement est empreint de souffrance dans laquelle se conjuguent à la fois le fait d'être l'enfant d'une mère malade mentale et le fait de vivre entre deux familles. Plusieurs se sont retrouvés dans la position parentifiée de celui qui se soucie et non de celui dont on se préoccupe. Tous ont conscience de la précarité de leur environnement et des défaillances parentales mais semblent partagés entre le refus d'identification et la trahison. Les relations avec leurs parents sont difficiles, plusieurs ne connaissent pas leur père. Deux jeunes filles dont les deux parents présentaient des pathologies mentales graves ont su préserver leur espace de vie partagée entre la FA et leurs parents.

\section{DISCUSSION}

Les familles étudiées plusieurs années après la sortie de 1'Unité de Soins avaient en commun des troubles de la relation parents-enfants et des problèmes de mise en place du processus de parentalité. La catégorisation entre deux grands profils de familles s'avère justifiée, elle distingue des familles qui assument leurs fonctions parentales dans la totalité [18] de celles qui présentaient au moment des soins des défaillances parentales sévères et ont aujourd'hui recours à des médiations (AEMO, prises en charge à l'ASE).

La situation des familles a donc été appréhendée en confrontant les données du passé et celles du présent. Ne disposant pas encore de modèle évaluatif pour cette démarche, nous avons construit nos propres outils de recherche: entretiens semi- 
directifs, questionnaires standardisés et subtests, ces deux derniers permettant des comparaisons intergroupes. Le fonctionnement de l'Unité de Soins étant spécifique, il ne s'agit pas d'une recherche randomisée. L'étude d'un groupe de comparaison prévu (enfants repérés par la PMI pour lesquels une proposition de prise en charge par l'Unité n'avait pas eu lieu) n'a pu être effectuée.

Trent huit familles répondaient à des critères précis, les uns correspondant aux prises en charge (soins avant l'âge de 18 mois d'une durée supérieure à un an), les autres à l'âge des enfants (nés entre 1985 et 1990) afin d'avoir un recul d'au moins 5 ans lors de 1'étude. Trente et une situations ont été analysées : 1) 24 dyades parentsenfants ont été rencontrées, 2) dans cinq cas, seule la mère a été interviewée et 3) pour sept jeunes non rencontrés (refus actif ou induit par les parents, situation de crise), on disposait d'au moins deux sources d'information. Le nombre de situations évaluées (31/38) indique un taux élevé de participation, à mettre en lien avec la permanence du personnel soignant et du secrétariat de l'institution. Les sept familles non rencontrées avaient été suivies moins longtemps à l'Unité ; les données recueillies auprès des professionnels qui se sont occupées d'elles ultérieurement nous amènent à penser que ces non répondants ne modifient pas l'ensemble des résultats.

Aujourd'hui, hormis une situation d'échec (enfants devenus Pupilles de l'Etat), les parents assument de façon totale ou partielle l'exercice, l'expérience ou la pratique de leur parentalité. Quant les enfants ont été placés, des relations parents-enfant régulières et authentiques ont été notées. La situation socio-familiale des familles s'est améliorée : stabilité des couples parentaux, développement des relations familiales avec la parentèle, investissement de la scolarité des enfants, capacité à rechercher de 1'aide si nécessaire... Parmi celles à haut risque psychique, les parents qui ont le mieux accepté les indications de soins de l'Unité et qui sont les plus vigilants envers leurs enfants sont 
ceux qui sont restés en relation avec un soignant ou un membre d'une équipe sociale. Ces relations dans la durée ont évité des mauvais traitements ou leur récidive et les enfants ne vivent plus dans un climat de violence Ces résultats vont dans le sens de plusieurs recherches évaluatives de programmes de soins précoces dans lesquelles les auteurs insistent sur l'importance de l'adhésion aux soins par les mères et la présence d'un conjoint aidant $[11,30]$.

L'hypothèse d'un développement « dans la norme » des enfants suivis à l'Unité est vérifiée. Les deux tiers des jeunes poursuivent une scolarité normale, ce qui implique un certain contrôle de soi et l'acceptation des normales sociales. Nous avons noté que les antécédents psychiatriques des parents ne sont pas forcément associés à un trouble mental chez le jeune, alors que la continuité de troubles psychiatriques a été noté dans des études épidémiologiques [31]. En début de prise en charge, deux enfants présentaient des défenses autistiques, l'un a évolué favorablement et l'autre, sorti de l'autisme, fréquente un hôpital de jour. Cependant, nous avons noté, comme le montrent plusieurs travaux de recherche $[27,35]$, un maintien de certain troubles de comportement (troubles de la conduite, hyperactivité, difficultés d'attention et de concentration, angoisse de séparation et tendance à l'anti-socialité). Le taux de troubles de comportement de $35 \%$ (11/31), significativement plus important chez les garçons, reste plus élevé que le taux de 12\% généralement admis en épidémiologie [20]. L'âge des jeunes (plus de la moitié de ceux rencontrés sont encore dans la période de préadolescence) et le type de population suivie (dépression maternelle, maladie mentale) explique cet écart. Toutefois, cette proportion de difficultés comportementales reste inférieure à ce qui est observé dans de nombreuses études sur des familles ayant de tels risques $[5,12,17,18,24]$ et à celui observé dans une étude sur les effets à la préadolescence des psychothérapies brèves [6] 
Parmi les limites et difficultés de ce type de recherche, on a noter la diversité des profils des familles, des modalités de soins et de leur durée. Dans cette étude, on retrouve néanmoins les liens classiques entre QI, scolarité et milieu social éducatif, de même les corrélations entre scolarité et compétences relationnelles avec les pairs ainsi que troubles de comportement et compétences relationnelles ${ }^{4}$. Il n'a pas été noté de différences entre les quatre groupes de jeunes en ce qui concerne les relations avec leurs pairs, de même pour le taux de troubles de comportement (tableau III). Ceci ne signifie d'ailleurs pas qu'il n'existe pas de différences cliniques (anti-socialité dans le groupe CAR, tendance à l'hyperactivité dans le groupe PSYK). La taille des groupes invite à une certaine prudence quant à l'amplitude de cet effet [29].

Soulignons, parmi les résultats notables, que les subtests verbaux sont toujours supérieurs au subtest de performance (tableau III, sauf dans le groupe CAR). Nous avons repéré chez un grand nombre de jeunes une facilité d'expression verbale. Certains ont pris du recul par rapport à la maladie et aux comportements parentaux, d'autres sont toujours sensibles aux fluctuations de l'état mental maternel. Au cours des entretiens, plusieurs ont montré leur capacité d'identification à des personnes diverses de leur entourage [34].

L'impact de soins très précoces est notable parmi 15 jeunes des familles « à risque » (la seizième ayant été placée à l'ASE à la fin des soins). Les enfants suivis avant l'âge d'un an ont de meilleures compétences que ceux pris en charge ultérieurement. Ainsi, neuf jeunes parmi les 12 pris en charge très tôt ont des compétences socio-relationnelles, aucun parmi les trois suivis à un an et plus ne

${ }^{4}$ Corrélations : 1 ) entre QI et scolarité ( $\mathrm{r}$ de Spearman $\left.=-0.79, \mathrm{p}<0.0001\right) ; 2$ ) entre compétences relationnelles avec les pairs et scolarité $(r=-0,57, p<0.002)$ ou existence de troubles de comportement $(r=-0,59, p<0,002)$. 
présente de telles compétences ; ces jeunes suivis très tôt ont également moins troubles du comportement et d'échec scolaire grave.

Ces résultats d'ensemble sont à mettre en relation avec l'accompagnement de longue durée de l'Unité de Soins et au travail en réseau effectué avec les autres institutions. Ces soins ont joué un rôle médiateur par rapport aux facteurs de risque psychosociaux quant au développement des enfants [29, 33]. On peut ainsi parler de prévention par le soin précoce du bébé, de la relation parent-enfant et de la parentalité. La proximité du lieu de soins est une donnée importante dans l'alliance thérapeutique par rapport aux familles et aux équipes soignantes. Après la sortie de l'Unité, plusieurs enfants et leurs parents ont pu effectuer une demande vers d'autres lieux de soins comme le Centre Alfred Binet (même immeuble que l'Unité); de même quant nous les avons rencontrés, plusieurs jeunes ont entrepris une démarche dans ce sens.

En l'absence d'un véritable groupe de comparaison et eu égard aux faibles effectifs des groupes étudiés, l'analyse reste descriptive, la méthodologie permet seulement d'évoquer des tendances quant à l'impact des soins proposés. Ce travail préliminaire souligne la nécessité de construire des procédures d'évaluation clinique standardisées et de développer des études longitudinales [10]. L'approche présentée ici ne met pas en évidence les effets des soins sur les structurations individuelles. Cependant, étant donné la richesse du matériel recueilli, plusieurs types d'analyses seront effectuées ultérieurement, permettant d'approfondir la question du fonctionnement psychique des jeunes.

\section{CONCLUSION}

Dès 1993, le rapport sur les aspects psychosociaux de la santé mentale des femmes de l'OMS mentionnait la nécessité de mieux connaître l'efficience des traitements, leur 
efficacité dans la rupture avec la chronicité institutionnelle et les négligences graves. C'est ce qui a été tenté dans cette étude sur le devenir de familles ayant bénéficié de soins précoces et prolongés. Les soins ont aidé les adultes à développer et maintenir leurs compétences parentales et leurs ressources personnelles. En particulier, pour les familles à haut risque, outre l'intérêt d'intervenir tôt et de moduler les soins en fonction des étapes maturatives du petit enfant et de son ou ses parents, la proximité des lieux de soins est un facteur aidant pour faciliter les contacts avec ces parents et leurs enfants et entre les intervenants médico-sociaux.

Dans cette recherche de type prospectif, nous avons pu évaluer les situations familiales mais les portraits de quelques jeunes seront à redéfinir après l'adolescence, période de questionnement identificatoire et identitaire. La prédictivité est toujours complexe; restent posées les questions du devenir de ces jeunes, celle de leur prise d'autonomie à l'âge adulte et de leur engagement futur dans l'expérience de la parentalité.

\section{Remerciements}

La recherche a été financée par la Fondation de Rothschild (convention n²578DH17F), la Fondation de France, la Fondation Grancher et la Fondation Mac Donald. Les auteurs tiennent à remercier le Dr. Françoise Jardin ancien médecin-directeur de l'Unité de Soins Spécialisés du XIIIè de Paris pour son aide dans l'élaboration de ce travail ainsi que 1'ensemble des soignants dont Marie-Françoise Pain, infirmière psychiatrique, qui a participé à la recherche des familles. Des équipes sociales de l'ASE de Paris et des référents de plusieurs PF ont également collaboré à l'étude. Nos remerciements s'adressent également à Luce Cohen, psychologue au CHRS de Cachan, à Daniel Ruffin, démographe, vacataire de recherche à 1'INSERM U.502 et à la Fondation de Rothschild, qui ont participé au recueil des données et à l'analyse clinique et statistique, ainsi qu'au Dr. Martine Lamour pour sa lecture attentive de ce manuscrit. 


\section{Références}

[1] Barnard KE, Magyary GS, Booth CL, Mitchell SK, Spieker SJ. Prevention of parenting altercations for woman with low social support. Psychiatr $1988 ; 51: 248-253$.

[2] Berthier M, Baraud C, Daban M, Paget A, Hay MC, et al. Evaluation de la prise en charge psychosociale en maternité : expérience du CHU de Poitiers. Arch Ped 1995;2 : 840-847.

[3] Bettschart W, Bolognini M, Plancherel B, Nunez R, Leidi C. Evènement de vie et santé psychique à la préadolescence : rôle du support social. Neuropsychiatr Enfance Adolesc $1992 ; 40: 421-430$.

[4] Boekholt M. Symptômes, santé, évolution chez 100 bébés: Un regard longitudinal sur le devenir somatique et psychique de 100 bébés en consultation de Médecine Préventive : étude clinique et statistique, Neuropsychiatr Enfance Adolesc 1988 ; 36 : 381-389.

[5] Cantwell DP, Baker L. Parental mental illness and psychiatric disorders in «at risk» children. J Clin Psychiat 1984 ; 45 : 503-507.

[6] Cramer B, Robert-Tissot C, Rusconi Serpa S. Du bébé au préadolescent, une étude longitudinale. Paris : Odile Jacob ; 2002.

[7] David M, Jardin F. Protéger et traiter les bébés exposés à des défaillances parentales, Devenir $1993 ; 5: 19-33$.

[8] Dugnat M. (sous la dir.) Troubles relationnels père-mère-bébé : quels soins ? Ramonville Saint Agne : Erès ; 1996.

[9] Dumaret A.C. Soins médico-sociaux et accompagnement psycho-éducatif. Revue de la littérature étrangère. Arch Ped $2003 ; 10,448-461$.

[10] Dumaret A.C. Evaluation de prises en charge précoces et interventions médico-sociales auprès de familles à risque. Analyse d'une démarche exploratoire. In Santé mentale du jeune enfant : prévenir et intervenir. Haddad A., Guédeney A., Greacen T. (sous la dir), Ramonville Saint Agne : Erès ; 2004, pp.127-137.

[11] Egeland B, Jacobvitz D, Sroufe LA. Breaking the circle of abuse. Child Dev $1988 ; 59$ : 080-1088.

[12] Ferrari P, Botbol M, Sibertin-Blanc D, Payant C, Lachal C, Presme N et al. Etude épidémiologique sur la dépression maternelle comme facteur de risque dans la survenue d'une psychose infantile précoce. Psychiatr Enf 1991 ; 34 : 35-97.

[13] Fraiberg S. Fantômes dans la chambre d'enfants. Paris : PUF ;1999.

[14] Goldberg M, Leclerc A Eds. Cohorte GAZEL. 20000 volontaires d'EDF/GDF pour la recherche médicale, Bilan 1989-1993. Paris : Inserm Eds ;1994.

[15] Grégoire J. Evaluation de l'intelligence de l'enfant. Bruxelles : Mardaga, 2 $2^{\text {ème }}$ éd ; 1995.

[16] Grégoire J. L'évaluation clinique de l'intelligence de l'enfant. Théorie et pratique du WISC-III. Bruxelles; Mardaga ; 2000.

[17] Hammen C, Gordon D, Burge D. Maternal affective disorders, illness and stress : risk for children's psychopathology. Am J Psychiatr 1987 ; 144 : 736-741.

[18] Hay DF, Pawlby S, Sharp D, Asten P, Mills A, Kumar R. Intellectual problems shown by 11-year-old children whose mothers had postnatal depression. J Child Psychol Psychiat 2001:871-889.

[19]Houzel D. (sous la dir.) Les enjeux de la parentalité. Ramonville Saint Agne : Erès ; 1999.

[20] Inserm, Expertise collective. Troubles mentaux. Dépistage et prévention chez l'enfant et l'adolescent. Paris : Inserm Eds ; 2002. 
[21] Jardin F, Rottman H Séparation thérapeutique d'un nourrisson de mère psychotique. Neuropsychiatr Enfance Adolesc $1986 ; 10:$ 447-454.

[22] Jardin F, Haab C, Montes de Oca M, Bresch D, Letronnier P, Moreau A. Dépression maternelle et troubles somatiques du bébé. Psychiatr Enf 1996 ; 39 : 103-136.

[23] Kashani JH, Canfield LA et al. Perceived family and social support : impact on children. J Am Acad Child Adolesc Psy 1994, 33 : 819-823.

[24] Keller MB, Beardslee WR, Dorer DJ, Lavori PW, Samuelson H, Klerman GR. Impact of severity of parental affective illness on adaptative functioning and psychopathology in children. Arch Gen Psychiatr 1986 ; 43 : 930-937.

[25] Lamour M, Barraco M. Perturbations précoces des interactions parents-nourrisson et construction de la vie psychique : Le jeune enfant face à une mère psychotique. Psychiatr Enf $1995 ; 38: 529-554$.

[26] Lieberman AF, Weston DR, Pawl JH. Preventive intervention and outcome with anxiously attached dyads, Child Dev $1991 ; 62: 199-209$.

[27] McGee R, Partridge F, William S, Silva P.A. A twelve-year Follow-Up of preschool hyperactive children. J. Am Acad Child Adolesc Psychiatry $1991 ; 30$ : 2, 224-232.

[28] Matthey S. P<.05, mais est-ce cliniquement significatif? Exemples pratiques pour les cliniciens. Devenir $2000: 12: 81-94$.

[29] Murray L. The socioemotional development of 5-year-old children of depressed mothers mothers. J Child Psychol Psychiat 1999 ; 40 : 1259-1271.

[30] Olds DL, Henderson CR, Kitzman H. Effects of prenatal and infancy nurse home visitation on surveillance and child maltreatment. Pediatrics $1995 ; 3: 365-372$.

[31] Radke-Yarrow M. Young children of affective ill parents : a longitudinal study of psychosocial development. J Am Acad Child Adolesc Psychiatry $1992 ; 31$ : 68-77.

[32] Rutter M, Quinton D. Parental psychiatric disturbance : effects on children. Psychol Med $1984: 14: 853-880$.

[33] Shaw DS, Vondra, JI, Hommerding KD, Keenan K, Dunn M. Chronic family adversity and early child behavior problems : A longitudinal study of low income families. J Child Psychol Psychiatr 1994 ; 35 : 1109-1122.

[34] Sroufe LA. Relationship, self, and individual adaptation. In Sameroff AJ, Emde RN Eds. Relationships disturbances in early child hood. New York Basic Books ; 1989.

[35] Stevenson J, Richman N, Graham PJ. Behaviour problems and language abilities at three years and behavioural deviance at height years, J Child Psychol Psychiat $1985 ; 26: 215$ 230 .

[36] Stoléru S, Moralès-Huet M. Psychothérapies mère-nourrisson dans les familles à problèmes multiples, Paris : PUF ; 1989. 
Tableau I : Profils des enfants suivis

\begin{tabular}{|c|c|c|c|c|}
\hline & HAND $(n=8)$ & PAR $(n=8)$ & CAR (7) & PSYK (n=9) \\
\hline \multicolumn{5}{|l|}{ âge d'admission } \\
\hline moyenne (mois) & $9,9 \pm 4,9$ & $8,8 \pm 5,2$ & $8,9 \pm 4,4$ & $6,8 \pm 5,4$ \\
\hline intervalles & {$[5-16]$} & {$[0,5-15]$} & {$[4-18]$} & {$[0,5-14]$} \\
\hline \multicolumn{5}{|l|}{ classification $0-3$} \\
\hline axe I: & 1 dépression nourrisson & 1 dépression nourrisson & 1 dépression nourrisson & 2 dépression nourrisson \\
\hline \multirow[t]{4}{*}{ diagnostic primaire } & 1 risque repli autistique & 1 tr. envahissants & 1 tr. multip. domaines & 2 tr. régulation \\
\hline & 1 anorexie précoce & 1 tr. régulation & 1 tr. régulation & 3 tr. attachement \\
\hline & & & 1 tr. expression émotion. & \\
\hline & & & 2 tr. attachement & \\
\hline
\end{tabular}

Axe II :

Troubles de la

3 R. anxieuse

3 R. anxieuse

2 R. anxieuse

relation

4 R. sous-impliquée

2 R. sous-impliquée

3 R. sous-impliquée

2 R. sous-impliquée

1 R. sur-impliquée

2 R. sur-impliquée

1 R. sur-impliquée

2 Tr. Mixtes

1 Tr. Mixtes

4 Tr. mixtes

1 R. hostile

axe III:

$\begin{array}{llll}\text { affections médicales } & 1 \text { trisomie } 8+\text { synd. Robin } & 1 \text { autisme infantile } & 1 \text { SAF et spina-bifida } \\ \text { tr. Développement } & 1 \text { réa. + hémiplégie } & \text { précoce } & 1 \text { SAF ? } \\ & 1 \text { synd Werdnig-Hoffman* } & 13 \text { hospitalis./1 an } \\ & 4 \text { prématurés** }\end{array}$

\section{axe IV:}

stress

Aucun

un

tous

tous

psychosociaux

modéré

sévères $>$ modérés

modérés > sévères

durée des soins

moyenne (mois)

$42,3 \pm 22,3$

$37,9 \pm 20,1$

$70,4 \pm 14,4$

$73,4 \pm 24,8$

intervalles

[18 - 77]

$[13-76]$

[49 - 84]

$[22-101]$

âge à l'évaluation

moyenne (annnées)

$12,3 \pm 1,6$

$12,3 \pm 1,5$

$13,2 \pm 0,9$

$13,3 \pm 1,5$

intervalles

$[10-14]$

$[10-15]$

$[11-14]$

[11 - 14]

Note au tableau 1: ( ) nombre d'enfants; SAF : syndrome d'alcoolisation foetale; * : amyotrophie spinale, ** dont un avec une maladie de Little, et un avec hémorragie cérébrale ; R. : relation, Tr. : troubles 
Tableau II : Scores aux subtests et QI estimé dans les groupes d'enfants

\begin{tabular}{|l|c|c|c|c|}
\hline \multicolumn{1}{|c|}{ Groupes testés } & QIG estimé & Vocabulaire & Similitudes & Cubes \\
\hline HAND (5) & $100,6 \pm 12,4$ & $11,6 \pm 3,5$ & $11,6 \pm 3,3$ & $8,6 \pm 4,7$ \\
PAR (8) & $104,0 \pm 18,1$ & $12,5 \pm 3,2$ & $11,6 \pm 3,2$ & $9,3 \pm 4,2$ \\
CAR (3) & $95,7 \pm 17,7$ & $8,0 \pm 2,6$ & $8,7 \pm 2,5$ & $10,3 \pm 3,5$ \\
PSYK (7) & $81,4 \pm 11,7$ & $9,4 \pm 2,1$ & $8,6 \pm 1,3$ & $5,3 \pm 2,5$ \\
Total (23) & $95,3 \pm 17,2$ & $10,8 \pm 3,2$ & $10,3 \pm 2,9$ & $8,0 \pm 4,0$ \\
$\quad p$ (analyse de variance) & $p<0,05$ & $n s$ & $n s$ & $n s$ \\
\hline
\end{tabular}

note $1: 3$ enfants du groupe HAND (très handicapés) et 1 enfant du groupe PSYK (conditions de passations non valides) n'ont pas été inclus dans les calculs.

note 2: A titre comparatif, QI moyen et catégories socio-professionnelles \{14]: cadres supérieurs et cadres moyens $(\mathrm{m}=110-111)$, ouvriers et personnels de service $(\mathrm{m}=96)$

note 3 : pour les 4 groupes, les différences sont statistiquement significatives entre chacun des deux subtests verbaux et le subtest de performance (test de Wilcoxon, différence Similitudes-Cubes $p<0,03$, différence Vocabulaire-Cubes $p<0,01$ ) 
Tableau III : Comportements socio-scolaires et compétences relationnelles avec les pairs

\begin{tabular}{|c|c|c|c|c|c|}
\hline \multirow{2}{*}{$\begin{array}{l}\text { Groupes d'enfants } \\
\text { HAND }\end{array}$} & \multicolumn{4}{|c|}{ Rutter * } & \multirow{2}{*}{\begin{tabular}{|l} 
Compétences $* *$ \\
Relations/pairs \\
$3,0 \pm 0,7(5)$
\end{tabular}} \\
\hline & $10,0 \pm 8,4(7)$ & $0,7 \pm 1,1$ & $2,1 \pm 2,5$ & $1,9 \pm 2,3$ & \\
\hline PAR & $11,0 \pm 6,8(8)$ & $1,3 \pm 1,0$ & $1,8 \pm 1,8$ & $1,9 \pm 1,6$ & $3,0 \pm 1,1(8)$ \\
\hline CAR & $10,1 \pm 4,4(7)$ & $2,3 \pm 1,3$ & $1,7 \pm 1,0$ & $1,3 \pm 2,1$ & $2,7 \pm 0,8(6)$ \\
\hline PSYK & $12,1 \pm 5,5(9)$ & $1,4 \pm 1,8$ & $2,7 \pm 1,9$ & $3,1 \pm 2,0$ & $3,0 \pm 0,5(8)$ \\
\hline total & $10,96,1(31)$ & $1,4 \pm 1,4$ & $2,1 \pm 1,8$ & $2,1 \pm 2,1$ & $2,9 \pm 0,8(27)$ \\
\hline test de Kruskal-Wallis & $\overline{n s}$ & $p<0,5$ & $n s$ & $n s$ & $N S$ \\
\hline sexe masculin & $15,5 \pm 4,2(12)$ & $2,0 \pm 1,7$ & $3,1 \pm 1,8$ & $3,2 \pm 2,0$ & $2,6 \pm 1,0(11)$ \\
\hline sexe féminin & $8,0 \pm 5,4(19)$ & $1,1 \pm 1,1$ & $1,4 \pm 1,6$ & $1,4 \pm 1,8$ & $3,1 \pm 0,5(16)$ \\
\hline test de Mann-Whitney & $p<0,001$ & Ns & $p<0,02$ & $p<0,02$ & Ns \\
\hline
\end{tabular}

* Questionnaire A Rutter

** compétences relationnelles avec les pairs : score établi comme suit : isolement, pas de copains (1), peu de compétences, liste de noms d'enfants, pas d'attachement (2), distinction entre amis et copains, lien privilégié avec certains (3), compétences importantes, visites régulières, activités communes (4). 